XLVIth Zakopane School of Physics, International Symposium Breaking Frontiers, Zakopane, Poland, May 16-21, 2011

\title{
Spectroscopy of Photosynthetic Pigment-Protein Complex LHCII
}

\author{
W.I. Gruszecki ${ }^{a, *}$, M. Zubik ${ }^{a}$, R. LuChowski ${ }^{a}$, W. Grudzinski ${ }^{a}$, Z. GryCZynski $^{b}$ \\ AND I. GRYCZYNSKI ${ }^{b}$ \\ ${ }^{a}$ Department of Biophysics, Institute of Physics, Maria Curie-Skłodowska University, Lublin, Poland \\ ${ }^{b}$ Centre for Commercialization of Fluorescence Technologies, University of North Texas \\ Health Science Center, Fort Worth, USA
}

\begin{abstract}
Light-harvesting pigment-protein complex of photosystem II is the most abundant membrane protein in the biosphere, comprising more than half chlorophyll molecules. The protein plays a role of photosynthetic antenna, collecting solar radiation and transferring excitations towards the reaction centers, where electric charge separation takes place. Efficient excitation energy capture and transfer requires unique organization of the complex and unique photophysical properties of the accessory pigments: chlorophylls and carotenoids. LHCII is also a place where extremely harmful singlet oxygen may be generated, under strong illumination conditions. Several physical mechanisms have been found in LHCII, operating to protect the photosynthetic apparatus against light-induced damage, including chlorophyll triplet and singlet excitations quenching by carotenoids. In this paper we discuss the results of our recent studies, carried out with the application of several molecular spectroscopy techniques (electronic absorption, fluorescence, resonance Raman and FTIR), designed to investigate molecular mechanisms responsible for regulation of excitation density in LHCII. Among the most interesting findings are the light-induced molecular configuration changes of the LHCII-bound xanthophylls, leading to conformational rearrangements of the protein. These mechanisms are discussed in terms of excessive excitation quenching in the pigment-protein complex subjected to overexcitation. Such an activity seems to represent a vital regulatory process in the photosynthetic apparatus, at the molecular level, protecting plants against photodegradation.
\end{abstract}

PACS: 87.64.- $\mathrm{t}$

\section{Introduction}

Light-harvesting pigment-protein complex of photosystem II (LHCII) is the largest photosynthetic antenna complex of plants, comprising photosynthetic accessory pigments: 8 molecules of chlorophyll $a, 6$ molecules of chlorophyll $b$ and 4 molecules of xanthophylls, 2 luteins, 1 neoxanthin and 1 violaxanthin $[1,2]$ (see Fig. 1). Efficient and fluent electric charge separation in the reaction center of Photosystem II requires accessory activity of several hundreds of photosynthetic pigments situated in the pigment-protein antenna complexes. Electronic excitation energy captured in the accessory pigment network is transmitted efficiently towards the reaction center.

On the other hand, overexcitation of the photosynthetic apparatus results in light-induced oxidative damage of the photosynthetic apparatus and entire photosynthesizing organism. Such a process is mediated by chlorophyll triplet excitations which can be formed via intersystem crossing from singlet excited states, under conditions of high excitation density exceeding capacity of the photochemical reactions. Regulation of the excitation density in the photosynthetic antenna systems is therefore a vital activity of plants, protecting them against light-induced damage. There are several mechanisms of such a regulation, active at different organization

\footnotetext{
* corresponding author; e-mail: wieslaw.gruszecki@umcs.pl
}

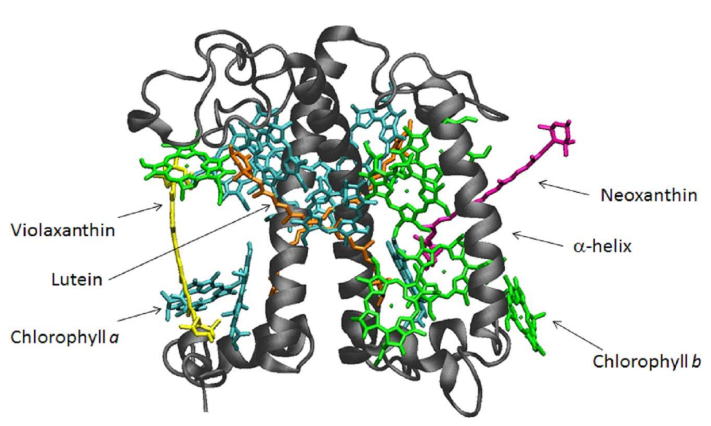

Fig. 1. Structure of LHCII. Main constituents of the complex are marked. The image was created with the visual molecular dynamics (VMD) software support. VMD is developed with NIH support by the Theoretical and Computational Biophysics group at the Beckman Institute, University of Illinois at UrbanaChampaign. LHCII coordinates were downloaded from the PDB database (PDB ID: 1RWT).

levels of plant organism, such as leaves, cells, chloroplast membranes and single pigment-protein complexes.

There are also different time scales on which such a regulation has to be realized, to follow both relatively slow and fast changes of sunlight intensity at the level of a plant and photosynthetic apparatus (see Fig. 2). The plant science has answered the questions regarding mechanisms of such a regulation at the level of entire organ- 


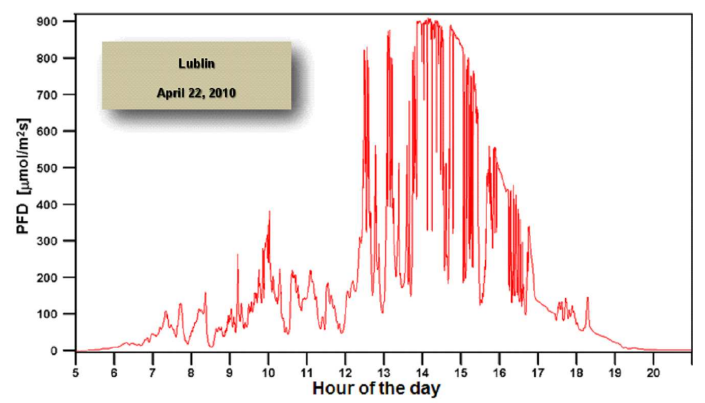

Fig. 2. Time dependence of photon flux density (PFD) of sunlight, recorded in Lublin (Poland) on April 22, 2010 .

isms (e.g. movements of leaves [3]) and at the cellular level (e.g. chloroplast phototranslocation [4]). The molecular mechanisms of regulation and photoprotection at the level of single photosynthetic pigment-protein complexes await to be resolved and are a subject of intensive research of multiple laboratories around the world. These researches resulted in several important findings, e.g. assignment of low-energy electronic states of chlorophylls in LHCII to protective excitation quenching [5], linking the light-induced geometric configuration changes of the LHCII-bound xanthophyll neoxanthin, with chlorophyll singlet excitation quenching by lutein [6] or involvement of xanthophyll cation radicals in photosynthetic antenna complexes in chlorophyll excitation quenching $[7,8]$. Below, we discuss the results of our recent spectroscopic studies on electronic excitation energy transfer and quenching in LHCII and on light-induced effects on organization of the complex.

\section{Light-induced spectral effects in LHCII}

Figure 3 presents the light-driven chlorophyll $a$ fluorescence quenching in LHCII. The effect has been originally observed by Jennings et al. [9] and was also analyzed by other groups [10-13]. Interestingly, as can be seen from Fig. 3, light-induced excitation quenching induced by blue light, absorbed both by chlorophylls and carotenoids, is much more effective that in the case of red light, absorbed exclusively by chlorophylls, despite equalized energy absorbed by LHCII in these two spectral regions. Moreover, the chlorophyll fluorescence lifetime analysis in LHCII shows that the lifetime distributions recorded with blue light are shifted towards lower values as compared to the distributions recorded with red light $[14,15]$. Such an observation suggests involvement of the LHCII-built xanthophylls in light-driven excitation quenching. In general, the average fluorescence lifetimes of chlorophyll $a$ in LHCII in the trimeric form are recorded in the range $3-4 \mathrm{~ns}[16]$. On the other hand, spontaneous fluorescence quenching in a single LHCII trimer can take place, as observed with application of the single molecule fluorescence spectroscopy [17].

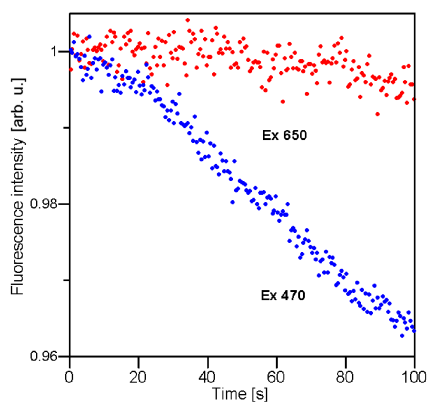

Fig. 3. Time course of chlorophyll $a$ fluorescence intensity in LHCII incorporated to liposomes. Fluorescence quenching was induced by illumination with red light (Ex $635 \mathrm{~nm}$ ) and with blue light (Ex $470 \mathrm{~nm})$, indicated. Light intensities have been set to adjust the same energy absorbed in both cases. Fluorescence intensity was normalized at time zero.

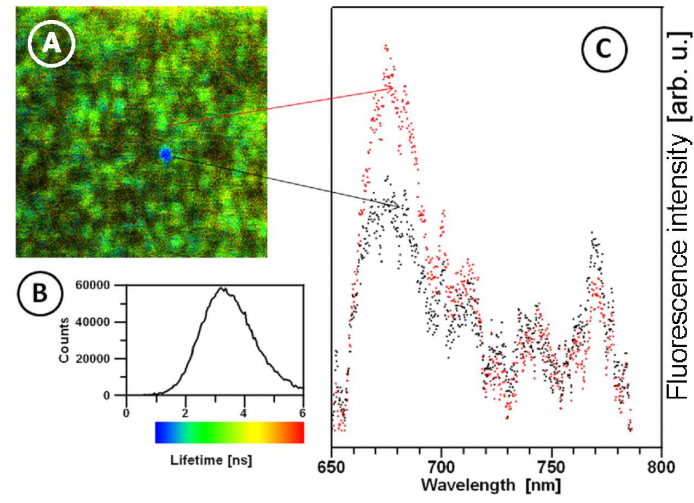

Fig. 4. (A) FLIM image of the $10 \times 10 \mu \mathrm{m}^{2}$ area of the glass slide covered with polylysine, at which the trimeric particles of LHCII were trapped from the micromolar suspensions in the buffer containing $0.04 \%$ detergent ( $\beta$-dodecyl maltoside). The image was recorded with illumination with the blue light laser $(470 \mathrm{~nm}$, $97 \mu \mathrm{W} / \mu \mathrm{m}^{2}$ ) and fluorescence emission was detected in the spectral region above $650 \mathrm{~nm}$. The repetition rate of the pulse laser was $20 \mathrm{MHz}$ and each pixel was scanned for $0.06 \mathrm{~ms}$. The image was scanned with resolution $300 \times 300$ pixels. (B) Fluorescence lifetime distribution corresponding to the image presented in part (A). (C) Fluorescence emission spectra recorded from the single particles imaged in part (A), indicated.

Figure $4 \mathrm{~A}$ presents the fluorescence lifetime imaging microscopy (FLIM) image of the sample constituted with trimeric LHCII. The histogram analysis of chlorophyll $a$ fluorescence lifetime distribution (Fig. 4B) shows that indeed, the average lifetimes recorded correspond to the trimeric complex. The FLIM image has been recorded with blue light $(470 \mathrm{~nm})$ which can additionally act as actinic light, driving molecular processes leading to excitation quenching. Detailed analysis of the image shows that some of the trimeric LHCII particles display fluorescence lifetimes considerably shorter than $3 \mathrm{~ns}$, displayed by blue color on the false color lifetime scale. The 
fluorescence emission spectra (Fig. 4C) of such particles are lower in intensity in the main emission band (650$700 \mathrm{~nm}$ ) but, interestingly, slightly higher fluorescence emission intensity can be observed in such a case, in the long-wavelength spectral region. Such an observation suggests that singlet excitation quenching in LHCII is associated with appearance of low-energy chlorophyll spectral forms. On the other hand, most effective excitation quenching is most probably associated with thermal energy dissipation. Indeed, as can be seen from Fig. 4C, the decrease in number of emitted quanta in the main fluorescence band is not compensated in the long-wavelength spectral region.

The fact that blue light, absorbed by carotenoids, plays a role of actinic light in the process of light-driven excitation quenching in LHCII allows to analyze possible light-induced effects in binding and/or geometrical configuration of xanthophylls by means of the resonance Raman scattering spectroscopy.

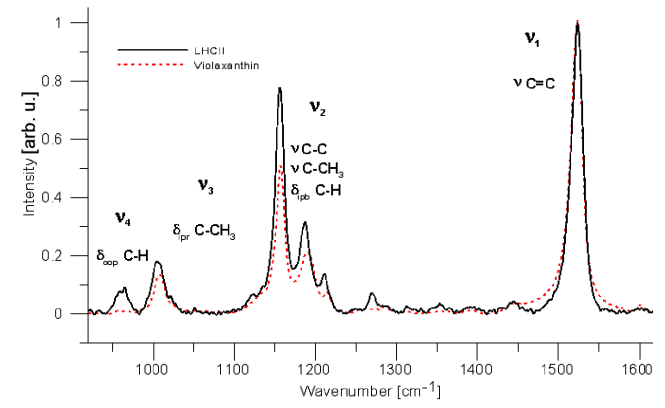

Fig. 5. Resonance Raman spectra of isolated violaxanthin and LHCII, indicated. The spectra were recorded with the $514.5 \mathrm{~nm}$ argon laser, power density of $11 \mu \mathrm{W} / \mu \mathrm{m}^{2}$. The spectra were normalized in the maximum of the $\nu_{1}$ band. Assignment of the main spectral bands to particular vibrations in carotenoid molecules is also presented.

Figure 5 presents the resonance Raman spectrum of xanthophylls in LHCII and the spectrum of isolated violaxanthin. As can be seen, a pronounced difference appears in the spectral region attributed to the carotenoid out of plane $\mathrm{C}-\mathrm{H}$ deformation vibrations, referred to as $\nu_{4}$ band. Owing to a relatively weak effect in the dipole transition, associated with this particular vibration, the $\mathrm{C}-\mathrm{H}$ deformation mode is Raman scattering silent, contrary to the IR absorption spectroscopy [18]. Interestingly, this particular band gains intensity after incorporation of xanthophylls into the pigment-protein complexes (see Fig. 5). Such an effect has been attributed to a twisting of the polyene chain, in the pigment binding site in LHCII [6]. Illumination of the complex results in further increase in the intensity of this band, in the case of neoxanthin, which was attributed to the light-driven twisting of the pigment [6]. Such an effect has been also observed in isolated chloroplasts and in intact leaves and has been correlated with the physiological response of the photosynthetic apparatus to light-stress conditions [6]. Inter- estingly, the resonance Raman study showed the opposite effect in the case of violaxanthin: the illumination of LHCII with strong light resulted in the light intensity-correlated decrease in the intensity of the $\nu_{4}$ band [19]. This effect has been interpreted in terms of a light-driven molecular configuration change, associated with uncoupling of violaxanthin from the protein [19]. Such a process, of violaxanthin transfer from the protein to the lipid phase of the thylakoid membrane, can play an important physiological function by making violaxanthin available for enzymatic de-epoxidation which takes place in the lipid phase [20]. De-epoxidation of violaxanthin in the photosynthetic apparatus of plants leads to accumulation of zeaxanthin and this process is particularly active under light stress conditions [20]. Xanthophylls which are present in the surrounding of LHCII, but not bound to the pigment binding sites, can modulate molecular organization of the protein $[21,22]$.

A lipid membrane system seems to be a model particularly interesting and well suited to study such a regulation owing to the specific localization and orientation of both the LHCII and xanthophylls which can diffuse freely in the membrane [23]. Polar xanthophylls, such as violaxanthin and zeaxanthin, have their hydroxyl groups located at the 3 and $3^{\prime}$ positions, at the opposite ends of the long, rod-like molecules. This determines both the localization and orientation of the xanthophyll molecules in the lipid bilayer: roughly vertical with respect to the plane of the membrane $[24,25]$. Polar xanthophylls, and in particular zeaxanthin, has been found to modify the supramolecular organization of LHCII in the lipid membrane system, which results in chlorophyll singlet excitation quenching manifested by decreased fluorescence emission intensity and by shortening of fluorescence lifetimes [23]. Supramolecular organization of proteins can be studied by analysis of infrared absorption spectra. In particular, the spectral region $1600-1700 \mathrm{~cm}^{-1}$, referred to as the "amide I" band, carries information on a secondary structure and organization of polypeptides [26].

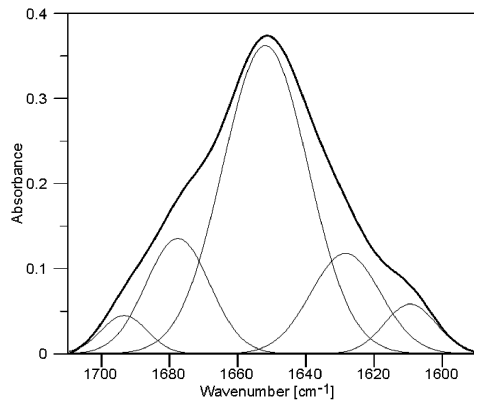

Fig. 6. FTIR absorption spectrum of LHCII, in the amide I region, presented along with the Gaussian analysis. The Gaussian components center at: $1609 \mathrm{~cm}^{-1}$, $1628 \mathrm{~cm}^{-1}, 1652 \mathrm{~cm}^{-1}, 1678 \mathrm{~cm}^{-1}$, and $1693 \mathrm{~cm}^{-1}$.

Figure 6 presents analysis of the amide I band of LHCII. The principal component centered at $1652 \mathrm{~cm}^{-1}$ 
represents the $\alpha$-helical structures of the protein and the second intensive component $\left(1678 \mathrm{~cm}^{-1}\right)$ represents the turns-and-loops structures of LHCII (see Fig. 1). Surprisingly, the analysis of the amide I band of LHCII reveals presence of the spectral components which can be attributed to $\beta$-sheets $\left(1628 \mathrm{~cm}^{-1}\right)$ and aggregated strands $\left(1710 \mathrm{~cm}^{-1}\right)$. The presence of these components can be interpreted as representing the intermolecular interactions of LHCII, leading to formation of molecular aggregates. Interestingly, illumination of LHCII with blue light, which results in light-induced molecular configuration changes of violaxanthin and neoxanthin and light-driven singlet excitation quenching, results also in a reversible, light-induced reorganization of the complex, manifested by changes in the structure of the amide I spectral band [15]. The main effect of illumination on the infrared absorption spectra of LHCII can be attributed to formation of intermolecular hydrogen bonds at expense of the turns and loops structures [15].

\section{Conclusions}

Isolated LHCII, subjected to illumination, is a model suitable to study physical mechanisms of photoprotection in the photosynthetic apparatus under light stress conditions, owing to the fact that isolated antenna protein has no possibility to transfer excitation energy out of it, towards a reaction center. The same circumstances can be expected in LHCII in plants under overexcitation conditions. The results of the studies carried out with application of molecular spectroscopy techniques, let hypothesize that photoprotection at the molecular level, in the photosynthetic antenna complex LHCII, is based on a following sequence of events: light-induced geometric configuration changes of the LHCII-bound xanthophyll(s) $\rightarrow$ changes in the supramolecular organization of the pigment-protein complex $\rightarrow$ chlorophyll singlet excitation quenching. The model proposed does not contradict parallel operation of alternative mechanisms which can be relevant from the physiological standpoint, as protecting photosynthetic apparatus against overexcitation-induced damage.

\section{Acknowledgments}

The research on molecular mechanisms of photoprotection at the molecular level was financed by the Ministry of Science and Higher Education of Poland from the funds for science in the years 2008-2011 within the research project N N303 285034 .

\section{References}

[1] Z. Liu, H. Yan, K. Wang, T. Kuang, J. Zhang, L. Gui, X. An, W. Chang, Nature 428, 287 (2004).

[2] R. Standfuss, A.C.T. van Scheltinga, M. Lamborghini, W. Kuhlbrandt, EMBO J. 24, 919 (2005).
[3] J. Ehleringer, I. Forseth, Science 210, 1094 (1980).

[4] H. Gabrys, Acta Physiol. Plant. 26, 473 (2004).

[5] Y. Miloslavina, A. Wehner, P.H. Lambrev, E. Wientjes, M. Reus, G. Garab, R. Croce, A.R. Holzwarth, FEBS Lett. 582, 3625 (2008).

[6] A.V. Ruban, R. Berera, C. Ilioaia, I.H. van Stokkum, J.T. Kennis, A.A. Pascal, H. van Amerongen, B. Robert, P. Horton, R. van Grondelle, Nature 450, 575 (2007).

[7] T.K. Ahn, T.J. Avenson, M. Ballottari, Y.C. Cheng, K.K. Niyogi, R. Bassi, G.R. Fleming, Science 320, 794 (2008).

[8] N.E. Holt, D. Zigmantas, L. Valkunas, X.P. Li, K.K. Niyogi, G.R. Fleming, Science 307, 433 (2005).

[9] R. Jennings, F. Garlaschi, G. Zucchelli, Photosynth. Res. 27, 57 (1991).

[10] V. Barzda, R.C. Jennings, G. Zucchelli, G. Garab, Photochem. Photobiol. 70, 751 (1999).

[11] W. Grudzinski, Z. Krupa, M. Garstka, W. Maksymiec, T.E. Swartz, W.I. Gruszecki, Biochim. Biophys. Acta 1554, 108 (2002).

[12] W.I. Gruszecki, W. Grudzinski, M. Matula, P. Kernen, Z. Krupa, Photosynth. Res. 59, 175 (1999).

[13] W.I. Gruszecki, P. Kernen, Z. Krupa, R.J. Strasser, Biochim. Biophys. Acta 1188, 235 (1994).

[14] W.I. Gruszecki, R. Luchowski, M. Zubik, W. Grudzinski, E. Janik, M. Gospodarek, J. Goc, Z. Gryczynski, I. Gryczynski, J. Plant. Physiol. 167, 69 (2010).

[15] W.I. Gruszecki, M. Zubik, R. Luchowski, W. Grudzinski, M. Gospodarek, J. Szurkowski, Z. Gryczynski, I. Gryczynski, J. Plant Physiol. 168, 409 (2011).

[16] B. van Oort, A. van Hoek, A.V. Ruban, H. van Amerongen, FEBS Lett. 581, 3528 (2007).

[17] T.P.J. Kruger, V.I. Novoderezhkin, C. Ilioaia, R. van Grondelle, Biophys. J. 98, 3093 (2010).

[18] B. Robert, P. Horton, A.A. Pascal, A.V. Ruban, Trends Plant Sci. 9, 385 (2004).

[19] W.I. Gruszecki, M. Gospodarek, W. Grudzinski, R. Mazur, K. Gieczewska, M. Garstka, J. Phys. Chem. B 113, 2506 (2009).

[20] P. Jahns, D. Latowski, K. Strzalka, Biochim. Biophys. Acta 1787, 3 (2009).

[21] W.I. Gruszecki, W. Grudzinski, M. Gospodarek, M. Patyra, W. Maksymiec, Biochim. Biophys. Acta 1757, 1504 (2006).

[22] A.V. Ruban, D. Philip, A.J. Young, P. Horton, Biochemistry 36, 7855 (1997).

[23] W.I. Gruszecki, M. Zubik, R. Luchowski, E. Janik, W. Grudzinski, M. Gospodarek, J. Goc, L. Fiedor, Z. Gryczynski, I. Gryczynski, Chem. Phys. 373, 122 (2010).

[24] W.I. Gruszecki, in: Carotenoids. Physical, Chemical and Biological Functions and Properties, Ed. J.T. Landrum, CRC Press, London, 2010, p. 19.

[25] W.I. Gruszecki, K. Strzalka, Biochim. Biophys. Acta 1740, 108 (2005).

[26] L.K. Tamm, S.A. Tatulian, Q. Rev. Biophys. 30, 365 (1997). 